\title{
New Nanocompoite Thermal Interface Materials Based on Graphene Flakes, Mesoscopic Microspheres and Polymers
}

\author{
Alex A Dmitriev, Alex S Dmitriev ${ }^{\mathrm{a}}$, Inna Mikhailova \\ NanoThermal Physics Laboratory, National Research University «Moscow Power Engineering Institute» \\ Russia, 111250, Moscow, Krasnokazarmennaya, 14
}

\begin{abstract}
In recent years, there has been a great interest in the development and creation of new functional energy materials, including for improving the energy efficiency of power equipment and for effectively removing heat from energy devices, microelectronics and optoelectronics (power micro electronics, supercapacitors, cooling of processors, servers and data centers). In this paper, the technology of obtaining new nanocomposites based on mesoscopic microspheres, polymers and graphene flakes is considered. The methods of sequential production of functional materials from graphene flakes of different volumetric concentration using epoxy polymers, as well as the addition of monodisperse microspheres are described. Data are given on the measurement of the contact angle and thermal conductivity of these nanocomposites with respect to the creation of thermal interface materials for cooling devices of electronics, optoelectronics and power engineering.
\end{abstract}

\section{Introduction}

Graphene research has attracted increasing interest from researchers in physics, materials science, chemistry, etc. Graphene consists of an unusual single-layer honeycomb lattice of $\mathrm{C}$ atoms, which differs from all other materials of this element. More specifically, the $\mathrm{C}$ atoms of graphene are $\mathrm{sp}^{2}$-bonded and densely packed in a crystal lattice. This closely resembles the basic building block found in graphitic materials of different dimensionalities. It has been demonstrated that graphene exhibits some special characteristics, such as a high electron mobility of up to $230000 \mathrm{~cm}^{2} / \mathrm{V} \cdot \mathrm{s}$ at low temperatures, an exceptional thermal conductivity approaching 5000 $\mathrm{W} / \mathrm{m} \cdot \mathrm{K}$ and superior mechanical properties with a large Young's modulus, exceeding 1.0 TPa the latter being comparable to those of single-walled nanotubes and even diamonds. With these intriguing physical and chemical properties, it is not surprising that graphene has been intensively investigated to exploit its functions as a promising material for future applications.

Graphene nanocomposites, in which graphene flakes are dispersed in a matrix (either polymer, ceramic or metal), have found numerous applications (energy, electronics and optoelectronics) that demand low weight with good mechanical as well as thermal performance [16]. The performance of composites is often limited by poor coupling between graphene flakes and matrix. For example, thermal transport through a composite can be dominated by thermal resistance at the interface that results primarily from phonon scattering due to mismatched phonon spectra in the two phases (graphene

\footnotetext{
a Corresponding author: asdmitriev@mail.ru
}

flakes and matrix) and from interfacial defects. The resistance is further increased by process-induced interfacial irregularities such as voids and interfacial stress, which result from graphene flakes surface roughness, poor adhesion between graphene flakes and matrix and dissimilarities in thermal expansion coefficient between graphene flakes and the surrounding matrix. Aside from the possibility of improving the overall mechanical properties of the composite, improved graphene flakes - matrix coupling would reduce interfacial thermal resistance and enhance heat conduction. Such an enhancement would have positive implications for a variety of applications, ranging from aircraft skins to lightweight heat exchangers.

In many cases, when discussing graphene thermal applications, we use the term graphene even when the actual material consists of a mixture of single layer graphene (SLG), bilayer graphene (BLG), and few-layer graphene (FLG). The latter is because for thermal applications the difference between SLG and FLG is not as important as for electronic applications. It is sometimes difficult to distinguish between FLG and graphite films or between FLG and graphite nanoplatelets $(\mathrm{GnP})$ or graphite nanoflakes $(\mathrm{GnF})$ used in composite materials. The definition of SLG - a single atomic plane of $\mathrm{sp}^{2}$-bound carbon - is strict.

It is important to note the possibility of using such hybrid nanocomposites as functional energy materials, for example, for lithium-ion batteries or materials with phase transitions. Development of high-power-density batteries, e.g., Li-ion batteries, enabled progress in mobile communications, consumer electronics, and 
automotive industries $[7,8]$. Temperature rise beyond the normal operating range negatively affects Li-ion battery performance. If overheated, the battery can suffer thermal runaway, cell rupture or explosion. A conventional approach for thermal management of high-powerdensity-ion battery packs is based on the utilization of thermal phase change materials (PCMs). They reduce the temperature rise in the battery due to the latent heat storing and phase changes over a small temperature range $[9,10]$. In this work, we will demonstrate new types of hybrid nanocomposites based on graphene flakes and metallic monodisperse microspheres. We investigate contact angles on the surface of such composites and measure the heat conductivity of several types of hybrid nanocomposites.

\section{Material and methods}

In the work, hybrid functional materials were studied in relation to energy problems, made of metal microspheres, polymer (epoxy resin) and graphene flakes. The method of obtaining composite materials is as follows. Graphene flakes produced by Graphene Star Ltd were used to prepare a nanocolloid solution (nanofluid), for which graphene flakes of various volume were added to pure distilled water and stirring was conducted for 20-30 minutes in a rotating centrifuge. Graphene flakes with a size of 5-10 microns with 3-10 layers of graphene (fewlayer graphene, FLG) were obtained. After that, the microspheres from $\mathrm{Sn}$ and $\mathrm{Er}_{3} \mathrm{Ni}$ (production of the Moscow Power Engineering Institute) were placed in a Petri dish and a nanocolloid solution of graphene flakes was poured into it. After that, the Petri dish was placed on the heater and the water from it was evaporated at different temperatures (from 60 to $100{ }^{\circ} \mathrm{C}$ ). After evaporation of the water, the microspheres covered with graphene flakes were heated to a temperature of $250{ }^{\circ} \mathrm{C}$ for 1-1,5 hours, which led to the effect of quenching the microspheres with graphene flakes on the surface. A general view of the microspheres covered with graphene flakes after drying and the quenching effect is shown in Figure 1. It is not difficult to see that the microspheres were very homogeneous, with a good coating of graphene flakes. At the same time, the adhesion value of graphene flakes to metal microspheres was very high, which made it possible to mix microspheres without destroying the graphene coating.
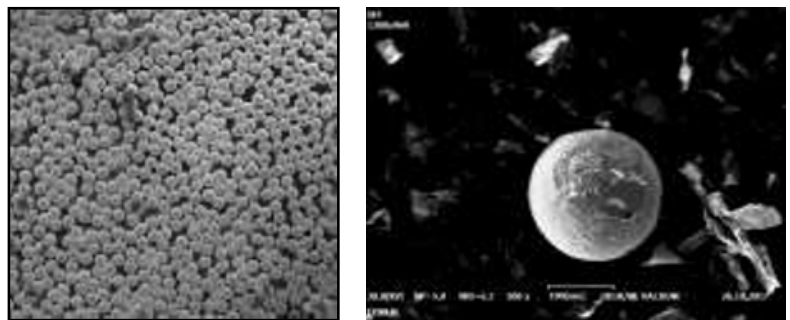

Figure 1. Microspheres Sn covered with graphene flakes after drying and the quenching effect

The structure of graphene nanocomposites is shown in the figure 2. Inside the composite, the metal microspheres are regularly located, the thickness of the coating of the microspheres by graphene flakes depends on their volume fraction, but does not exceed 20-25 $\mu \mathrm{m}$.

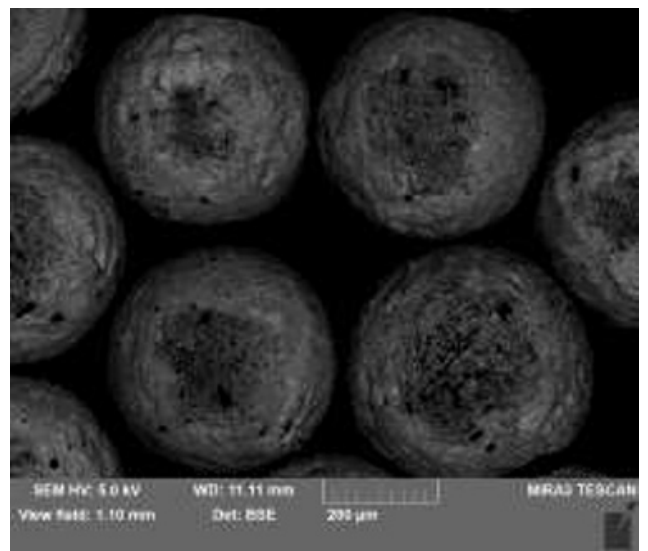

Figure 2. Structure of graphene nanocomposites (SEM)

In addition to hybrid nanocomposites, tablets were made from graphene flakes of various thicknesses at various pressures. The production takes place in two stages: 1) the choice of a certain volume of graphene powder, on which the thickness of the obtained samples depends; 2) loading the selected volume of powder into the press for further pressing. The choice of a certain volume of powder is carried out by a conventional $20 \mathrm{ml}$ medical syringe. To do this, the end of the syringe is carefully cut along the first line of the calibration. As a result, a large hole is obtained equal to the diameter of the syringe, with which it is already possible to easily obtain the required volume of graphene powder. The collected volume of graphene is pushed out by the plunger of the syringe into the mold, which is closed with a plug on top. Then, the press-fitting parameters are selected on the control panel of the press and then the process is started. At the end of the process, a cap is opened and a mold with a received graphene tablet is lifted using the control buttons. For this work, samples of graphene tablets were made at various press parameters and various powder volumes.

The measurement of quantity was carried out in volumetric units with the help of a syringe, which allowed to measure a certain amount and was convenient for loading the metered powder into the mold. Since this press is a highly specialized equipment, intended mainly for metallographic research, it constantly heats the material loaded in the mold. Therefore, the lowest possible temperature of $150{ }^{0} \mathrm{C}$ was used for manufacturing. As it was said above, 3 samples were prepared for each pressure value with powder volumes equal to 2,5 and $10 \mathrm{ml}$ respectively.

Different values of pressure and volume gave different thicknesses of the obtained samples (Figure 3).

In some experiments, isolated flakes were homogeneously dispersed in epoxy matrix and almost no large agglomerates or bundles were observed. The strong interfacial adhesion between oxygenic groups on the of $\mathrm{GnF}$ and epoxy molecular chains led to high embedding and tight binding of $\mathrm{GnF}$ in the matrix. For this reason, these wrinkled and isolated GNPs effectively prevents direct contact between graphite nanoparticles in the 
composite, which give advantages for forming a large microcapacitor network and suppressing the leakage current.

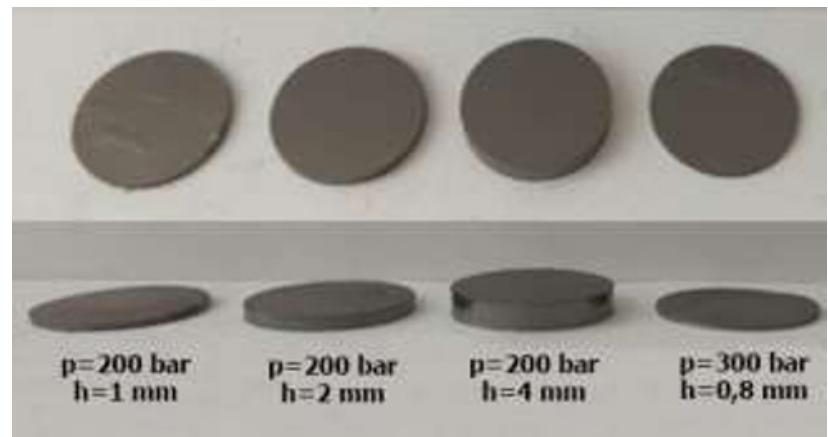

Figure 3. Samples of tablets from graphene flakes

\section{Results and discussion}

First, we studied the dynamic contact angles and evaporation rates of drops of distilled water on graphene tablets, which were obtained by pressing at various pressures (200 and 300 bar) of graphene flakes (Figure 4). Various properties of hybrid nanocomposites based on microspheres and graphene were investigated. In some cases, microspheres coated with graphene were placed in a polymer matrix. For the experiments, tablets were prepared in the form shown in Figure 3. For all types of samples, contact angles and thermal conductivity were studied. To determine the angle of wetting of drops on the surface of samples of a hybrid nanocomposite, Kruss EasyDrop measuring system was used with special heater and temperature control system.

Investigations of the electrical resistance of various samples were carried out under pressure variations during their preparation. Table 1 shows the results of these measurements.

Table 1. Electrical conductivity of graphene tablets

\begin{tabular}{|c|c|c|}
\hline \#Tablets & $\begin{array}{c}\text { Thicknes } \\
\mathbf{s}(\mathbf{m m})\end{array}$ & $\begin{array}{c}\text { Specific electrical } \\
\text { resistance }(\mathbf{O h m} / \mathbf{~ m})\end{array}$ \\
\hline $\mathrm{P}=200$ bar & 4 & 1375 \\
\hline $\mathrm{P}=300$ bar & 3.8 & 789.47 \\
\hline
\end{tabular}

With an increase in pressure by $50 \%$, the resistance dropped to $57 \%$. This result is not accidental. As the pressure increases, the number of bonds for the passage of current along the sample increases. Thus, it can be argued that denser samples have a higher electrical conductivity.

We also investigated the rates of evaporation of droplets of distilled water on the surface of graphene tablets at different temperatures (Figure 5). To heat the substrates, the ITO was used, the measurements were made by a non-contact infrared thermometer and thermocouples.

Investigation of thermal conductivity of graphene hybrid nanocomposites includes experiments on the measurement of thermal conductivity by two methods: the method of thermal resistance of a layer and the method of laser flash (LFA-laser flash analysis) - Figure 6. The laser flash method is a more accurate method, so the results of these methods will be compared with each other. Measurements of the thermal conductivity of hybrid nanocomposites were carried out for dilute samples.

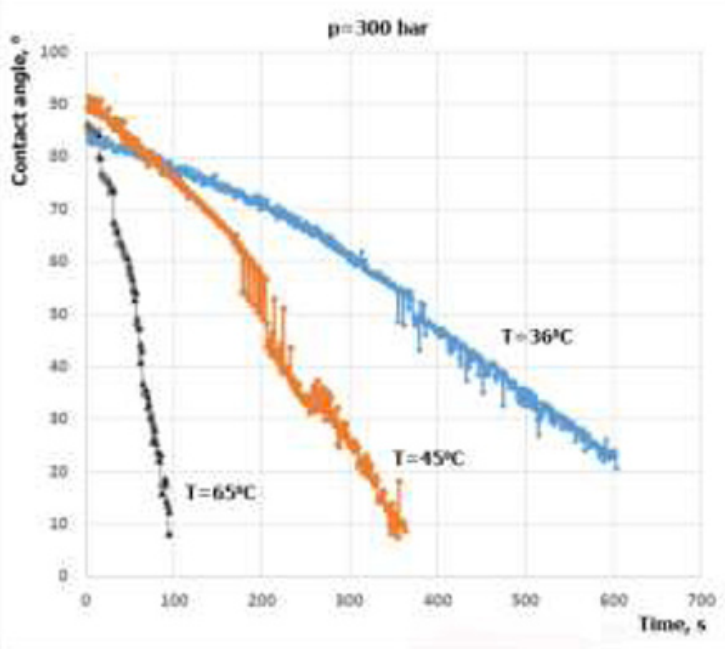

Figure 4. Changing the contact angle with time

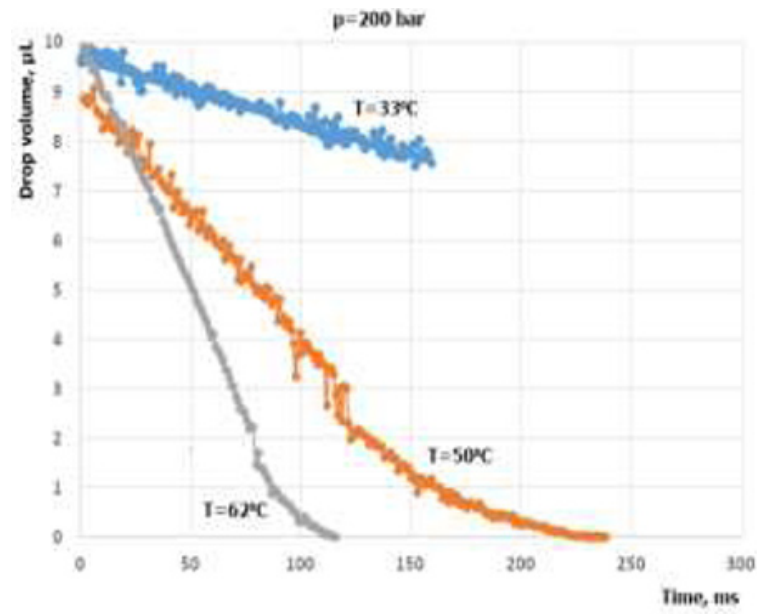

Figure 5. Evaporation rate of distilled water droplets on the graphene tablet surface

In the first series of experiments, samples based on a freely lying layer of microspheres coated with graphene flakes were investigated. In the second series of measurements, the thermal conductivity of densely packed microspheres coated with multilayer graphene was studied (Figure 2). It is important to note that the dependence of thermal conductivity on the volume fraction of graphene flakes has a sharp increase with a volume fraction of about $2.5-3 \%$ (Figure 7). Thus, the graphene layers near the microspheres play an important role in the process of heat transfer - with increasing amounts of graphene flakes, thermal conductivity sharply increases. This fact can be used to control heat transfer in various nanocomposites, in particular, used as thermal interface materials. The results of the thermal conductivity show that there is an optimal volume fraction of graphene flakes in the range 2,5 - 10\%. For large values of the volume fraction, the thermal conductivity practically does not increase. 


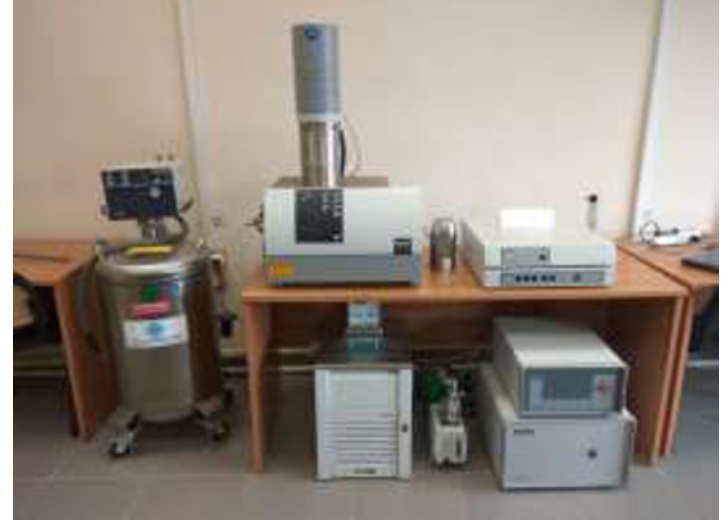

Figure 6. The measuring complex by the laser flash method NETZSCH LFA 457 MicroFlash

Apparently, physically this means that the observed percolation in this range of the mating fraction and the further increase in the amount of graphene flakes does not affect the mechanism of phonon heat transfer. The following series of experiments were carried out on the samples shown in Figure 8.

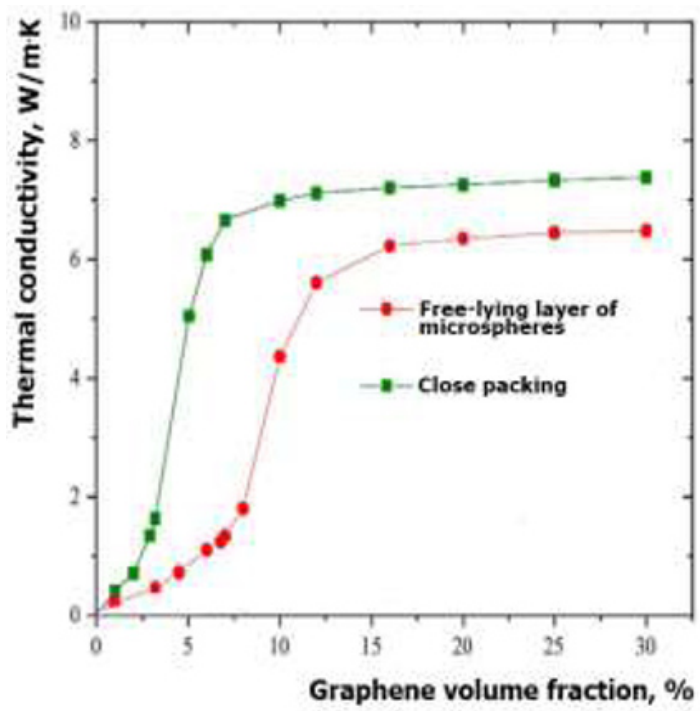

Figure 7. Thermal conductivity of microspheres coated graphene flakes

These samples were hybrid nanocomposites - the inclusion of graphene flakes in the polymer matrix of the epoxy, as well as the hybrid nanocomposite microspheres + graphene flakes inside the epoxy matrix. The dependences of the thermal conductivity of such hybrid nanocomposites are presented in Figure 8. In addition, the thermal conductivity of a polymer matrix (epoxy resin) is shown here.

With increasing temperature, the thermal conductivity of all samples decreases, which is associated with an increase in phonon scattering at the boundaries of graphene flakes. It is important to note that the use of graphene coated microspheres greatly increases the thermal conductivity. This indicates the possibility of using hybrid nanocomposites with microspheres as functional energy materials.

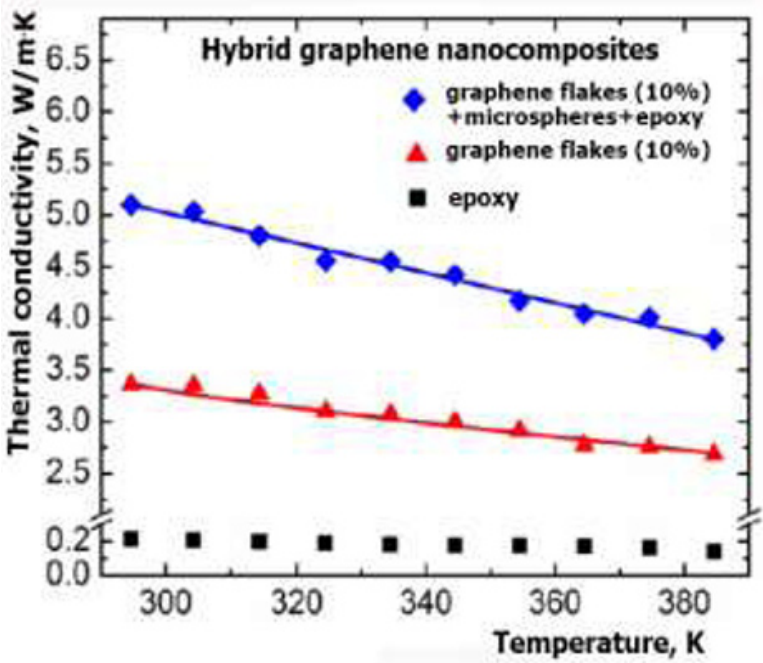

Figure 8. Thermal conductivity of hybrid nanocomposites

\section{Conclusions}

In this paper we presented the results of the properties of new hybrid nanocomposites based on graphene flakes, metal microspheres and epoxy polymers. The obtained data on the contact angles on the surface of such composites and their thermal conductivity at room temperature make it possible to draw conclusions about the potential use of these materials as functional energy materials (for example, as the thermal interface materials). In particular, for practical applications of graphene nanocomposites are outlined in an example using thermal phase change materials for thermal management of highpower battery packs.

\section{Acknowledgments}

The authors thank S Alekseev (Graphene Star Ltd, UK) for making graphene flakes. This work was supported by the Russian Science Foundation (project No. 17-1901757).

\section{References}

[1]. J. Renteria, D. Nika and A. Balandin, Appl. Sci. 4, 525-547 (2014)

[2]. A. Bar-Cohen, K. Matin and S. Narumanchi, J. Electronic Packaging 137 040803-1 (2015)

[3]. A. Dmitriev, Introduction to nanothermophysics, (BINOM Press, Moscow, 2015)

[4]. A. Roy, B Farmer, V Varshney et al., ACS Appl. Mater. Interfaces 4 (2) 545-563 (2012)

[5]. S A Alekseev, A S Dmitriev, P G Makarov and I A Mikhailova, Journal of Physics: Conf. Series. 891 012361 (2017)

[6]. F. Gardea, M. Naraghi and D. Lagoudas, ACS Appl. Mater. Interfaces 6 (2) 1061-1072 (2014).

[7]. A. Balandin, IEEE Spectr. 29 (2009) 35-39.

[8]. A.S. Dmitriev, A. R. Valeev, J. Phys.: Conf. Ser. 891 012359 (2017)

[9]. F.Agyenim, N. Hewitt, P. Eames and S. Mervyn, Renew Sustain. Energy Rev. 14 615-628 (2010)

[10]. V. Goyal and A Balandin, Appl. Phys. Lett. 100 073113 (2012) 